\title{
Efecto biológico y social de los factores asociados al riesgo cardiovascular en la población adulta joven, revisión documental
}

\author{
Biological and social effect of the factors associated with cardiovascular risk \\ in the young adult population, documentary review in the period 2010-2020
}

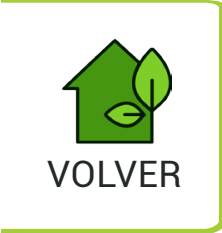

Rodríguez-López Danna María

Universidad Santiago de Cali

https://orcid.org/0000-0002-3306-660X

Gutiérrez-Escobar Alejandro

Universidad Santiago de Cali

https://orcid.org/0000-0002-8155-3277

Cortes-Gómez Nicolás

Universidad Santiago de Cali

https://orcid.org/0000-0001-8656-2343

Calero-Saa Pedro Antonio

Universidad Santiago de Cali

https://orcid.org/0000-0002-9978-7944

\begin{tabular}{|c|c|c|}
\hline Recepción/Submission: & $\begin{array}{c}\text { Evaluación de contenidos/ } \\
\text { Peer-review outcome: }\end{array}$ & Aprobación/Acceptance \\
\hline Abril (April) de 2019 & Mayo (May) de 2019 & Julio (July) de 2019 \\
\hline
\end{tabular}

Cómo citar.

Rodríguez-López, D. M; Gutiérrez-Escobar, A; Cortes-Gómez, N. y Calero-Saa, A. (2020). Efecto biológico y social de los factores asociados al riesgo cardiovascular en la población adulta joven, revisión documental. Revista Científica Sabia, 6(1). pp, 176-189. Doi: https://doi.org/10.47366/sabia. v6n1a12

\begin{abstract}
Resumen
Introducción: Las enfermedades cardiovasculares para la Organización Mundial de la Salud (OMS) representan uno de los más grandes problemas en materia de salud pública a nivel mundial, las cuales son consideradas como una de las primeras causas de mortalidad ocasionando 17 millones de muertes por año (1). Según estudios realizados, la población adulto joven tiende a encaminarse hacia la adquisión de conductas y estilos de vida poco saludables, debido a que el ámbito académico produce cambios en estas conductas con complicaciones, tales como, comer entre horas, alto consumo de comidas rápidas, poco tiempo para comer, disminución en la realización de actividad física e incremento de consumo de alcohol y tabaco (2). Objetivos: Conocer acerca de las variables relacionadas con los efectos biológicos y sociales de factores de riesgo cardiovascular en la población adulta joven. Materiales y métodos: se realizó una revisión documental de la literatura existente en el período 2010-2020 de acuerdo a los criterios de inclusión y exclusión propuestos. Resultados y conclusiones: Los resultados encontrados indican que los componentes sociales representan mayor influencia en el desarrollo de los factores de riesgo afectando directamente la dimensión biológica.
\end{abstract}

Palabras clave: Riesgo Cardiovascular; Obesidad; Tabaquismo; Consumo De Alcohol; Hábitos Alimenticios; Enfermedades Cardiovasculares; Adulto Joven. 


\begin{abstract}
Introduction: Cardiovascular diseases for the World Health Organization (WHO) represent one of the greatest public health problems worldwide, which are considered one of the leading causes of mortality, causing 17 million deaths per year (1). According to studies, the young adult population tends to move towards the acquisition of unhealthy behaviors and lifestyles, because the academic environment produces changes in these behaviors with complications, such as eating between meals, high consumption of fast foods, little time to eat, decrease in physical activity and increased consumption of alcohol and tobacco (2). Objectives: To know about the variables related to the biological and social effects of cardiovascular risk factors in the young adult population. Materials and methods: a documentary review of the existing literature in the period 2010-2020 was carried out according to the proposed inclusion and exclusion criteria. Results and conclusions: The results found indicate that the social components represent a greater influence on the development of risk factors, directly affecting the biological dimension.
\end{abstract}

Key words: Cardiovascular Risk; Obesity; Smoking; Alcohol Consumption; Eating Habits; Cardiovascular Diseases; Young Adult. 


\section{Combustión de carbón vegetal, enemigo latente del medio ambiente \\ Mary Lucero Valencia Cuero}

\section{Introducción}

Las enfermedades cardiovasculares para la Organización Mundial de la Salud (OMS) representan uno de los más grandes problemas en materia de salud pública a nivel mundial, las cuales son consideradas como una de las primeras causas de mortalidad ocasionando 17 millones de muertes por año (1).

Se define como factor de riesgo cardiovascular a aquella característica la cual puede ser tanto biológica como un hábito que contribuye al aumento de la posibilidad de tener o de fallecer a causa de una enfermedad cardiovascular en aquellas personas que los presenten. Estos factores son clasificados en aquellos no modificables (sexo, edad, factores genéticos o antecedentes familiares) y en aquellos modificables (Hipertensión arterial, hipercolesterolemia, diabetes mellitus, obesidad y tabaquismo) (3).

La OMS, estipula que el tabaquismo es uno de los factores de riesgo cardiovascular que es causa de muerte evitable con mayor importancia a nivel mundial, ya que el consumo del tabaco causa 3 millones de muertes o más al año teniendo repercusiones tanto sociales como económicas de gran importancia (4).

Según estudios realizados, la población adulto joven tiende a encaminarse hacia la adquisión de conductas y estilos de vida poco saludables, debido a que el ámbito académico produce cambios en estas conductas con complicaciones, tales como, comer entre horas, alto consumo de comidas rápidas, poco tiempo para comer, disminución en la realización de actividad física e incremento de consumo de alcohol y tabaco (2).

El presente estudio es de tipo documental en donde se buscó literatura existente en el período 20102020 de acuerdo a los criterios de inclusión y exclusión propuestos, con el objetivo de conocer acerca de las variables relacionadas con los efectos biológicos y sociales de factores de riesgo cardiovascular en la población adulta joven. Los resultados encontrados indican que los componentes sociales representan mayor influencia en el desarrollo de los factores de riesgo afectando directamente la dimensión biológica.

\section{Materiales y métodos}

Este estudio es una Revisión documental la cual se realizó con base a la evidencia disponible en torno a los estudios realizados durante el período de 2010 al 2020. La información de este estudio fue extraída de artículos de investigación resultantes de la búsqueda en bases de datos determinados por las palabras claves y la temática abordada en el mismo.

Posterior, se realizó una revisión y análisis bibliográfico de artículos relacionados con los efectos biológicos y sociales de los factores de riesgo cardiovascular, publicados en español e inglés, en los años 2010 hasta 2020, con el fin de recolectar información, indagando en diferentes bases de datos. Culminando con la creación de una base de datos en excel para el análisis y recopilación de la información.

Criterios de inclusión: Artículos que fueran estudios originales, artículos publicados desde el año 2010, artículos en diferentes idiomas y artículos completos.

Criterios de exclusión: Artículos que presenten falta de respaldo científico, información derivada de blogs o páginas no científicas, artículos de revisión de literatura o revisión sistemática. 


\section{Discusión de los resultados}

El proceso de búsqueda ésta se efectuó a través de combinaciones de las palabras claves: "Riesgo cardiovascular AND Adultos jóvenes, Obesidad AND Adultos jóvenes, Adulto joven AND Tabaquismo, Consumo de alcohol AND Riesgo cardiovascular, Hábitos alimenticios AND Adultos, Enfermedad cardiovascular AND Riesgo cardiovascular AND adulto, Tabaquismo AND Obesidad, Consumo de alcohol AND Tabaquismo" en diferentes bases de datos como pubmed, sciencedirect, scopus, y scielo. Estos estudios fueron enumerados en una matriz según título, objetivo de estudio, metodología utilizada, tamaño de la muestra, tipo de estudio, variables evaluadas y resultados relevantes (Ver tabla 1).

Después de la selección inicial de 143 artículos, fueron escogidos 50 artículos potencialmente relevantes publicados en el período 2010-2020 para la inclusión final, los cuales pasaron a través del proceso de lectura crítica con el propósito de verificar el cumplimiento estricto de criterios de inclusión, siendo estos remitidos para la extracción de datos en respuesta de los objetivos planteados por el presente estudio.

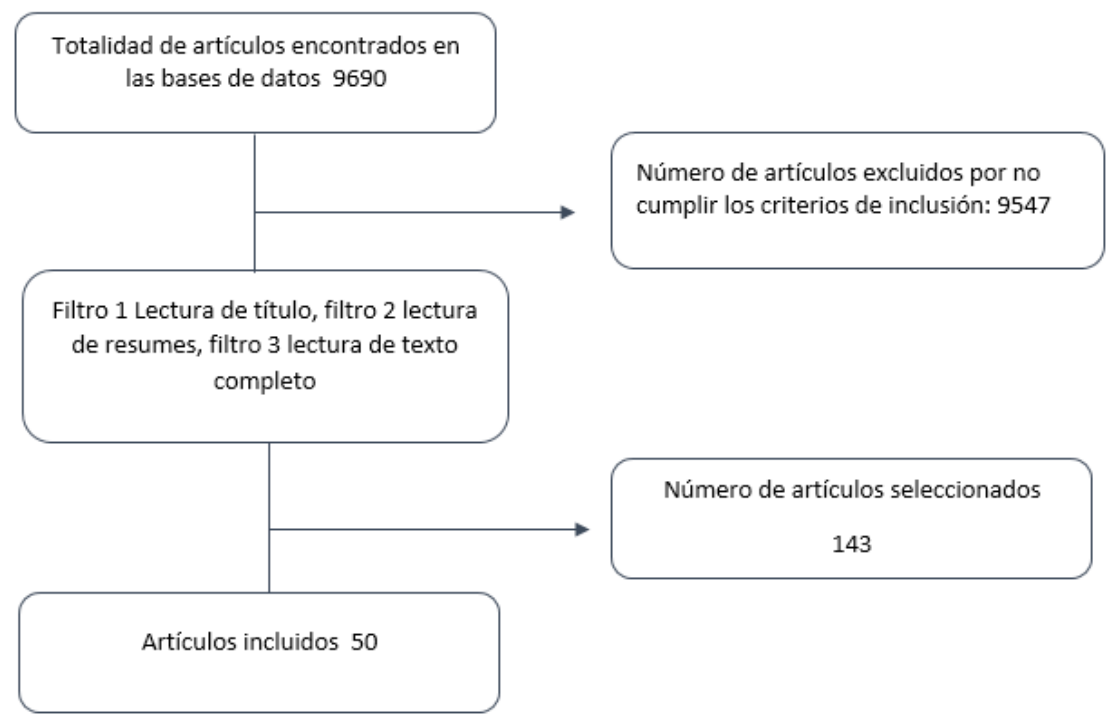

Figura 1. Diagrama Prisma del flujograma de selección de los artículos

La distribución por año en el que fueron publicados los 50 artículos seleccionados en la búsqueda documental es la siguiente: 2010:5,2011:5,2012:4,2013:1,2014:8,2015:6,2016:2,2017:8,2018:5,

2019:2,2020:4 (Ver ilustración 1). En cuanto al idioma, de los 50 estudios incluidos, el 80\%(40) fueron publicados originalmente en el idioma español, el 18\%(9) publicados en inglés y el $2 \%(1)$ en portugués (Ver ilustración 2). Según el tipo de estudio, los seleccionados se constituyeron por 24 estudios descriptivos, 21 estudios transversales, 2 estudios de cohorte, 1 estudio experimental, 1 estudio cuasi experimental y 1 estudio ecológico (Ver ilustración 3). Según el país de origen, los artículos seleccionados son de: México:8,Colombia:10,España:15,Chile:5,cuba:3,Uruguay:1,Peru:1, Ecuador. 2, Brasil: 4, Suecia: 1 (Ver ilustración 4).

\section{Factor de riesgo cardiovascular}

Un factor de riesgo cardiovascular (FRCV) es aquel que contiene una característica biológica, hábito o estilo de vida que puede aumentar la posibilidad de padecer o de fallecer a causa de una ECV. Dichos factores comprenden efectos biológicos refiriéndose al proceso y los mecanismos de producción de la 


\section{Combustión de carbón vegetal, enemigo latente del medio ambiente \\ Mary Lucero Valencia Cuero}

enfermedad; así como efectos sociales tales como los comportamientos, emociones y estado mental propios del individuo condicionados por el contexto social en el que se desenvuelve el mismo (91-93).

Los factores de riesgos que se han identificado en la población adulta joven se divide en dos grupos: el primero llamado factores modificables como lo son el sedentarismo, la obesidad, los hábitos alimenticios, el tabaquismo, el alcohol y el segundo llamado factores no modificables como lo son los trastornos de base genética, la edad (mayor de 45 años) y el sexo (predominio masculino) $(1,16,91,94,95)$.

\section{Sedentarismo}

El sedentarismo se establece como uno de los factores de riesgo más común para la adquisición de una ECV, siendo este; producto de un desequilibrio entre la ingesta de alimentos y el gasto calórico diario, relacionado con la tendencia al desarrollo de la obesidad, alcanzando altos índices de prevalencia siendo además un factor de riesgo supremamente significativo para el advenimiento de la enfermedad coronaria $(14,15)$.

En contraste el sexo masculino presenta un perfil metabólico con biomarcadores de resistencia a la insulina e inflamación más elevados, independientemente de la actividad física realizada. Las concentraciones elevadas de glucosa, triglicéridos y ácidos grasos libres en la circulación pueden generar un exceso de radicales libres y desencadenar una cascada bioquímica de inflamación, disfunción endotelial, hipercoagulabilidad y aumento de la actividad simpática (16-18).

Sotomayor et al., plantea una explicación a estas diferencias tan importantes en la prevalencia de falta de actividad física y conductas sedentarias puede encontrarse en los aspectos socioeconómicos que determinan las condiciones ambientales de los vecindarios (calles mal iluminadas, con alto índice de criminalidad, etc (19).

\section{Obesidad}

La obesidad se encuentra caracterizada por el incremento de la adiposidad que puede ser asociada a una alteración metabólica trayendo consigo misma una serie de comorbilidades tales como; hipertensión arterial, diabetes mellitus, entre otras, las cuales disminuyen su calidad de vida (20).

Silva, et al, encontró que el tejido adiposo produce varias adipocitocinas, como la interleucina-6 (IL6), la adiponectina, la leptina y el factor de necrosis tumoral (TNF- a), cuyo desequilibrio modifica varios factores asociados con enfermedades cardiovasculares (apetito, equilibrio energético, sensibilidad a la insulina, presión arterial, metabolismo de los lípidos, inmunidad y homeostasis) . La activación de estos elementos favorece el desarrollo de un proceso inflamatorio de baja intensidad, caracterizado por un ligero aumento de biomarcadores inflamatorios y oxidativos (PCR). Este aumento puede contribuir activamente a la aparición de lesiones endoteliales, lo que resulta en un factor de riesgo de enfermedad coronaria (21).

Álvarez, et al, Halló que en Perú, a nivel nacional existe una tendencia ascendente del sobrepeso y la obesidad a medida que se incrementa la edad, excepto durante la adolescencia y en adultos mayores. También, el sobrepeso y la obesidad afecta más a la población adulta y se asocia con vivir en la zona urbana y la condición de no pobreza. Por otro lado, se observó que ambos fueron más prevalentes en el área urbana y menos prevalente en el contexto rural. Lo cual se puede explicar que por la mayor urbanización y desarrollo económico conlleva a cambios de los estilos de vida, provocando modificaciones en los patrones de alimentación y la actividad física (22). 


\section{Hábitos Alimenticios}

En términos de efecto social, los hábitos alimentarios son fuertemente relacionados con características de tipo social, económico y cultural de una población determinada los cuales se han ido modificando según la interacción familiar y la situación económica. Aproximadamente la mitad de la población adulta joven no desayunan omitiéndolo uno o más días debido al poco tiempo que se tiene para desayunar o la falta de apetito debido a evaluaciones en ámbito estudiantil. Además, el consumo de frutas y verduras suele ser bajo. En esta población se destaca el consumo de bebidas azucaradas y artificiales. Adicional a lo anterior, se menciona que la dieta es fuertemente condicionada por el ámbito social del adolescente lo cual tiene un fuerte impacto en la imagen corporal y los comportamientos que adoptan frente a la misma dentro de un grupo de amigos, lo que es traducido a querer ser aceptados por su aspecto físico en el mismo (23) (106).

Además, en la infancia comienza a instaurarse ciertos hábitos alimenticios que se consolidan en la edad adulta; en la adolescencia e inicio de la juventud se ven influenciados por las preferencias personales, mayor independencia o imagen física. En varios de los casos suelen no desayunar, lo que es condicionado por el estrés de la vida universitaria o el tener una mayor independencia, en donde deben cocinar sus propios alimentos, desde la inexperiencia, a realizar recetas poco elaboradas con alto porcentaje de embutidos, enlatados o carnes pre-cocidas o simplemente omitir la comida. Por otro lado, se estipula también el hecho de tener ansiedad, producto de presentación de exámenes, lo que puede llevar a consumir mayor cantidad de productos poco saludables con el objetivo de saciarla (25).

\section{El Tabaquismo}

Según la evidencia encontrada el consumo de tabaco aumenta el riesgo de padecer un IAM (Infarto Agudo al Miocardio) o muerte súbita. Los mecanismos nocivos del tabaco son diversos, debido a sus efectos sistémicos, vasculares, así como protrombogénicos. El exceso en el consumo de cigarrillos genera disfunción endotelial en las arterias, aumentando la rigidez en la capa íntima y media modificando nocivamente el perfil lipídico $(26,27)$.

Diversos autores estipulan que este se asocia al consumo de tabaco con las alteraciones en el perfil lipídico: el nivel plasmático de c-HDL es más bajo, mientras que el c-LDL, colesterol total y triglicéridos son más altos, lo que ocasiona una mayor incidencia de enfermedad aterosclerótica en individuos fumadores. También se observó que la PAS y la PAD son significativamente más altas en los fumadores, favoreciendo la aparición de HTA $(26,28-30)$.

Según Hernández et al, el ser humano como ser social adquiere ciertos determinantes para su desenlace en el medio. Los motivos laborales, familiares, ansiedad y demás los hace más propensos a padecer este tipo de enfermedades. La adopción de este hábito es originado de la búsqueda de la identidad propia de los adolescentes iniciando a los 15 años los cuales son condicionados por sus amigos dentro de un grupo y la necesidad de pertenecer al mismo siendo este hábito el modo de ingresar (14).

\section{Consumo de alcohol}

El alcohol es una sustancia adictiva utilizada por los adultos jóvenes en mayor medida, los que potencialmente la utilizan son los estudiantes universitarios o aquellos que se encuentran próximos a recibir la educación superior lo cual ha generado mayor interés en la salud pública por sus evidentes índices de consumo. $(31,32)$.

Redondo Et al, con relación a el consumo de alcohol con las enfermedades cardiovasculares (ECV) está actualmente en discusión, ya que si bien hay pruebas de que pequeñas cantidades, inferiores 


\section{Combustión de carbón vegetal, enemigo latente del medio ambiente \\ Mary Lucero Valencia Cuero}

a $25 \mathrm{~g} /$ día, podrían ser un factor que redujera el riesgo de aparición de cardiopatía isquémica, ictus isquémico y diabetes tipo 2, algunos condicionantes pueden modificar este efecto (patrón de consumo, edad) (33).

Sin embargo, Noreys, et al, estipula que el consumo de alcohol en mayor medida a nivel del sistema cardiovascular presenta efectos que empeoran el funcionamiento de este ya que hay una reducción de la función contráctil del corazón manifestándose las arritmias cardiacas y esto es debido a un efecto inotrópico negativo del etanol sobre el músculo cardíaco, pero este efecto suele ocultarse por un efecto indirecto cronotrópico e inotrópico positivo secundario a la liberación de catecolaminas (34).

En la sociedad actual se reconoce al consumo de alcohol como una tendencia social y cultural, a la que la población juvenil tiene acceso y un acercamiento precoz. Se ha evidenciado que los motivos por el cual los estudiantes consumen drogas y bebidas alcohólicas en altas cantidades son: tener tiempo libre, amigos o alteraciones emocionales, conflictos familiares, o comportamientos éticos cuestionables. $(31,32)$.

Montero, et al, El consumo de alcohol constituye una de las toxicomanías más difundidas actualmente por su carácter legal y aceptación. Varios pueden ser los motivos que pueden conllevar al consumo de esta droga, pudiéndose mencionar: la baja autoestima, la tendencia al aislamiento y depresión, la impulsividad, las conductas rebeldes, los conflictos en el rol sexual y otros problemas de salud mental $(35,36)$.

\section{Trastornos de base genética}

Algunas funciones fisiológicas, como el metabolismo celular, la segregación y captación de hormonas, son definidas por la transducción de genes los cuales también presentan mutaciones como medida de adaptación al medio ambiente y condiciones de vida, los cuales serán codificados en el genoma siendo así heredados por las siguientes generaciones, dichos cambios pueden llevar a desarrollar enfermedades metabólicas, cardiovasculares, entre otras $(134,135)$.

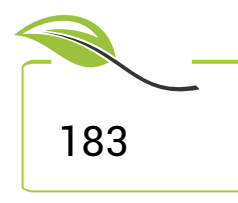

\section{Edad}

En términos de efecto biológico se estipula que la edad es un factor interno que potencia la probabilidad de tener una enfermedad cardiovascular lo que se traduce que a mayor edad, mayor riesgo de padecer una de estas enfermedades. Desde el punto de vista fisiológico, las personas con el pasar de los años presentan una disminución en la fuerza muscular, disminución visual, auditiva y cognitiva además de presentarse cambios estructurales en los vasos y en el corazón llevando a la adquisión de hipertensión arterial, que es considerada como otro factor de riesgo cardiovascular $(39,40)$.

Por otro lado, se mencionan que la gran mayoría de los procesos cardiovasculares se deben a la aterosclerosis la cual es una enfermedad que tiene sus inicios en la vida fetal, con un progreso en la niñez y en la adolescencia teniendo, en la vida adulta, un desarrollo acelerado. En esta patología se presenta un depósito o placa en el interior de las arterias la cual está conformada por colesterol, calcio y grasas. Esta se va endureciendo con el tiempo y causa un estrechamiento que es visible en el adulto mayor causando en ellos una limitación en el flujo sanguíneo que oxigena los tejidos corporales por ende puede crear coágulos que pueden viajar a diferentes estructuras desencadenando otras patologías como lo son el accidente cerebrovascular, cardiopatías isquémicas, aneurismas, entre otras (113). 


\section{El sexo (masculino)}

Según García M, et al, dentro los procesos biológicos que se encuentran presentes en el ser humano existen apreciables diferencias entre los hombres y las mujeres ligadas al sexo, esto se encuentra principalmente relacionado a la producción de hormonas que generan un impacto variable en la adquisición de FRCV o manifestación de las ECV. Lo anterior se encuentra relacionado a los beneficios que tienen los estrógenos en el sistema cardiovascular. El estrógeno, es señalado como el posible responsable de una cardioprotección, el cual regula la menstruación, disminuye la concentración de CLDL en grados variables según su relación con la progesterona, la cual es una posible razón por la que las mujeres en edad reproductiva son menos propensas a estas enfermedades (129).

Conclusión: Las características biológicas y sociales representan condicionantes para la relación entre los distintos tipos de factores de riesgo cardiovascular, simultáneo a la interacción entre el contexto de sus clasificaciones y dimensiones que implican al ser humano, llevándolo a la adopción de conductas desfavorable tanto benéficas para el cuidado de la salud. La Adultez joven es conocida como una etapa del ciclo vital en la cual estas conductas tenderán a formar gran influencia en los hábitos de vida, donde se identificó un marcado índice de conductas nocivas, factores de riesgo e incluso desarrollo precoz de enfermedades cardiovasculares y metabólicas, siendo predominantes factores modificables tales como el sedentarismo, dieta desbalanceada, índices de obesidad, consumo de alcohol y tabaquismo por lo cual es crucial el desarrollo de estrategias de promoción y prevención en las diferentes esferas de la salud en este o demás grupos poblacionales.

Agradecimientos: Agradecemos a la Universidad Santiago de Cali, y al docente Pedro Antonio Calero Saa por apoyo en el desarrollo de la presente investigación.

Declaración de conflicto de interés: Los investigadores no presentaron ningún tipo de conflicto de interés.

Financiación: Este estudio no ha sido financiado por ninguna institución, fue realizada con fines académicos. 


\section{Referencias}

Alvarado C, Jaramillo M, Matijasevic E, Rendón ID, Quitian J. Estudio poblacional de factores de riesgo cardiovascular relacionados con el estilo de vida, hallazgos electrocardiográficos y medicación actual de pacientes valorados por el servicio de Cardiología. Rev Colomb Cardiol. el 1 de febrero de 2012;19(2):61-71.

Alcivar JEA, Vera NAC, Marcillo WEP, García MP, Vásquez HEA. Riesgo cardiovascular antropométrico de estudiantes universitarios. Rev Cuba Cardiol Cir Cardiovasc. el 8 de marzo de 2020;26(1):921.

Álvarez-Dongo D, Sánchez-Abanto J, Gómez-Guizado G, Tarqui-Mamani C. Sobrepeso y obesidad: prevalencia y determinantes sociales del exceso de peso en la población peruana (2009-2010). Rev Perú Med Exp Salud Pública. septiembre de 2012;29:303-13.

Alcoholismo en jóvenes universitarios de diferentes carreras | [Internet]. [citado el 6 de junio de 2020]. Disponible en: https://www.psicologiacientifica.com/alcoholismo-jovenes-universitarios/

Areiza M, Osorio E, Ceballos M, Amariles P. Conocimiento y factores de riesgo cardiovascular en pacientes ambulatorios. Rev Colomb Cardiol. el 1 de marzo de 2018;25(2):162-8.

Batista ANR, García T, Franco EAT, Azevedo PS, Barbosa MF, Zornoff LAM, et al. Comparison of morphometry and ventricular function of healthy and smoking young people. BMC Cardiovasc Disord [Internet]. el 6 de febrero de 2020 [citado el 4 de junio de 2020];20. Disponible en: https://www.ncbi.nlm.nih.gov/pmc/ articles/PMC7006152/

Castaño-Perez G. A. Y Calderon-Vallejo G. A. Problems associated with alcohol consumption by university students. Rev Lat Am Enfermagem. octubre de 2014;22(5):739-46.

Carballosa Y. C., Zapata K. A., Luis L. M., Aguilar E. M., Pérez A del CR, Carbajal YP. Factores de riesgo de enfermedad cardiovascular en estudiantes de preuniversitario. Rev Cuba Med Gen Integral [Internet]. el 16 de diciembre de 2019 [citado el 4 de junio de 2020];35(4). Disponible en: http://www.revmgi.sld.cu/index. $\mathrm{php} / \mathrm{mgi} /$ article/view/844

Castro Cuesta JY, Abellán Huerta J, Leal Hernández M, Gómez Jara P, Ortín Ortín EJ, Abellan Aleman J. Estilos de vida relacionados con el riesgo cardiovascular en estudiantes universitarios. Clínica E Investig En Arterioscler. 2014;26(1):10-6.

Fisiopatología - EcuRed [Internet]. [citado el 31 de mayo de 2020]. Disponible en: https://www.ecured.cu/ Fisiopatolog\%C3\%ADa

Fernández de Bobadilla J, Sanz de Burgoa V, Garrido Morales P, López de Sá E. Riesgo cardiovascular: evaluación del tabaquismo y revisión en atención primaria del tratamiento y orientación sanitaria. Estudio RETRATOS. Aten Primaria. el 1 de noviembre de 2011;43(11):595-603.

Factores de Riesgo Cardiovascular: Síntomas, Tratamientos y Más [Internet]. [citado el 2 de septiembre de 2019]. Disponible en: https://cuidateplus.marca.com/enfermedades/enfermedades-vasculares-y-delcorazon/factores-de-riesgo-cardiovascular.html

Félix-Redondo FJ, Fernández-Bergés D, Palomo Cobos L, Buitrago Ramírez F, Pérez Castán JF, Lozano Mera L. Prevalencia de consumo de alcohol y factores de riesgo cardiovascular en un área sanitaria de Extremadura. Estudio Hermex. Aten Primaria. el 1 de abril de 2012;44(4):201-8.

Guerrero-R N, Yépez-Ch MC. Factores asociados a la vulnerabilidad del adulto mayor con alteraciones de salud. Univ Salud. junio de 2015;17(1):121-31.

García M. Factores de riesgo cardiovascular desde la perspectiva de sexo y género. Rev Colomb Cardiol. el 1 de enero de 2018;25:8-12.

Hernández-Escolar J, Herazo-Beltrán Y, Valero MV. Frecuencia de factores de riesgo asociados a enfermedades cardiovasculares en población universitaria joven. Rev Salud Pública [Internet]. octubre de 2010 [citado el 4 de septiembre de 2019];12:852-64. Disponible en: https://www.scielosp.org/article/ rsap/2010.v12n5/852-864/ 
Hernández-Escolar J, Herazo-Beltrán Y, Valero MV. Frecuencia de factores de riesgo asociados a enfermedades cardiovasculares en población universitaria joven. Rev Salud Pública [Internet]. octubre de 2010 [citado el 4 de septiembre de 2019];12:852-64. Disponible en: https://www.scielosp.org/article/ rsap/2010.v12n5/852-864/

Lobos Bejarano JM, Brotons Cuixart C. Factores de riesgo cardiovascular y atención primaria: evaluación e intervención. Aten Primaria. el 1 de diciembre de 2011;43(12):668-77.

León-Latre M, Moreno-Franco B, Andrés-Esteban EM, Ledesma M, Laclaustra M, Alcalde V, et al. Sedentarismo y su relación con el perfil de riesgo cardiovascular, la resistencia a la insulina y la inflamación. Rev Esp Cardiol. el 1 de junio de 2014;67(6):449-55.

Lavielle-Sotomayor $\mathrm{P}$, Pineda-Aquino $\mathrm{V}$, Jáuregui-Jiménez $\mathrm{O}$, Castillo-Trejo M. Actividad física y sedentarismo: Determinantes sociodemográficos, familiares y su impacto en la salud del adolescente. Rev Salud Pública. marzo de 2014;16(2):161-72.

Martínez-Quintana E, Rodríguez-González F, Medina-Gil JM, Garay-Sánchez P, Tugores A. Actividad de CYP2C19 y factores de riesgo cardiovascular en pacientes con síndrome coronario agudo. Med Clínica. el 20 de septiembre de 2017;149(6):235-9.

Martínez Pastor A, Balanza Galindo S, Leal Hernández M, Martínez Navarro A, Conesa Bernal C, Abellán Alemán J. Influencia del género en los estilos de vida que se asocian a enfermedades vasculares en Universitarios. Hipertens Riesgo Vasc. el 1 de julio de 2010;27(4):138-45.

Meseguer Zafra M, Rosa Guillamón A, García-Cantó E, Rodríguez García PL, Pérez-Soto JJ, Tárraga López PJ, et al. Influencia de un programa de ejercicio físico terapéutico en diferentes indicadores clínicos relacionados con la dislipidemia en sujetos adultos de 26 a 73 años con algún factor de riesgo cardiovascular. Hipertens Riesgo Vasc. el 1 de enero de 2019;36(1):21-7.

Morales G, Guillen-Grima F, Muñoz S, Belmar C, Schifferli I, Muñoz A, et al. Factores de riesgo cardiovascular en universitarios de primer y tercer año. Rev Médica Chile. marzo de 2017;145(3):299-308.

Martínez S MA, Leiva O AM, Sotomayor C C, Victoriano R T, Von Chrismar P AM, Pineda B S. Factores de riesgo cardiovascular en estudiantes de la Universidad Austral de Chile. Rev Médica Chile. abril de 2012;140(4):426-35.

Meléndez J, León S, León, Vázquez C, Barquera S, Hernández Nava L, et al. Hábitos alimentarios, actividad física y estilos de vida en adolescentes escolarizados de la Ciudad de México y del Estado de Michoacán Eating habits, physical activity and lifestyles among adolescents in Mexico City and the State of Michoacán. el 1 de enero de 2017;23.

Morales I G, del Valle R C, Soto V Á, Ivanovic M D. Factores de riesgo cardiovascular en estudiantes universitarios. Rev Chil Nutr. diciembre de 2013;40(4):391-6.

Núñez Rocha GM, López Enríquez I, Ramos Hernández SR, Ramos Peña EG, Guevara Valtier MC, González Treviño IM. Riesgo cardiovascular en pacientes de primer nivel de atención. Rev Salud Pública Nutr. el 8 de mayo de 2018;14(1):1-8.

Noreys Bárbaro OB, Annalie Elizabeth FP, Laura Beatriz SM, Miguel PR, RobertoFB. Principales consecuencias del etilismo crónico sobre el sistema cardiovascular. Hospital Lenin. Holguín, enero-octubre del 2018. En: I Congreso Virtual de Ciencias Básicas Biomédicas de Granma [Internet]. 2020 [citado el 6 de junio de 2020]. Disponible en: http://www.cibamanz2020.sld.cu/index.php/cibamanz/cibamanz2020/paper/view/531

Puig-Nolasco A, Cortaza-Ramírez L, Cristina Pillon S. Consumo de alcohol entre estudiantes Mexicanos de medicina. Rev Lat Am Enfermagem. junio de 2011;19(SPE):714-21.

(PDF) Fisiología del crecimiento [Internet]. ResearchGate. [citado el 15 de mayo de 2020]. Disponible en: https://www.researchgate.net/publication/232420740_Fisiologia_del_crecimiento

Rodríguez Domínguez L, Díaz Sánchez ME, Ruiz Álvarez V, Hernández Hernández H, Herrera Gómez V, Montero Díaz M. Factores de riesgo cardiovascular y su relación con la hipertensión arterial en adolescentes. 
Rev Cuba Med. marzo de 2014;53(1):25-36.

Ruano C, Lucumí E, Albán J, Arteaga S, Fors M. Obesity and cardio-metabolic risk factors in Ecuadorian university students. First report, 2014-2015. Diabetes Metab Syndr Clin Res Rev. el 1 de noviembre de 2018;12(6):917-21.

Ramos-Morales N, Marín-Flores J, Rivera-Maldonado S, Silva-Ramales Y. Obesidad en la población escolar y la relación con el consumo de comida rápida. Index Enferm. 2006;15(55):9-12.

Rodríguez-Artalejo F, Graciani A, Guallar-Castillón P, León-Muñoz LM, Zuluaga MC, López-García E, et al. Justificación y métodos del estudio sobre nutrición y riesgo cardiovascular en España (ENRICA). Rev Esp Cardiol. el 1 de octubre de 2011;64(10):876-82.

Sandrino VL, Meléndrez EH. Factores psicosociales relacionados con las enfermedades cardiovasculares. Rev Cuba Cardiol Cir Cardiovasc. el 1 de septiembre de 2016;22(3):152-6.

Soriano-Maldonado A, Cuenca-García M, Moreno LA, González-Gross M, Leclercq C, Androutsos O, et al. Ingesta de huevo y factores de riesgo cardiovascular en adolescentes; papel de la actividad física: Estudio HELENA. Nutr Hosp. junio de 2013;28(3):868-77.

Soriano-Maldonado A, Cuenca-García M, Moreno LA, González-Gross M, Leclercq C, Androutsos O, et al. Ingesta de huevo y factores de riesgo cardiovascular en adolescentes; papel de la actividad física: Estudio HELENA. Nutr Hosp. junio de 2013;28(3):868-77.

Vera-Remartínez EJ, Lázaro Monge R, Granero Chinesta S, Sánchez-Alcón Rodríguez D, Planelles Ramos MV, Vera-Remartínez EJ, et al. Factores de riesgo cardiovascular en adultos jóvenes de un centro penitenciario. Rev Esp Salud Pública [Internet]. 2018 [citado el 4 de septiembre de 2019];92. Disponible en: http://scielo.isciii.es/scielo.php?script=sci_abstract\&pid=S1135-57272018000100416\&lng=es\&nrm= iso\&tlng=es

Vernaza-Pinzón P, Villaquiran Hurtado A, Paz Peña C, Ledezma Chavez B. Riesgo y nivel de actividad física en adultos, en un programa de estilos de vida saludables en Popayán. Rev Salud Pública. el 1 de septiembre de 2017;19:624-30.

Vélez-Álvarez C, Gil-Obando LM, Ávila-Rendón CL, López-López A. Factores de riesgo cardiovascular y variables asociadas en personas de 20 a 79 años en Manizales, Colombia. Univ Salud. junio de 2015;17(1):32-46. 


\section{Rodríguez-López Danna Maria}

Universidad Santiago de Cali

Pregrado en fisioterapia. Correo: dannarodriguez-1@hotmail.com

Gutiérrez-Escobar Alejandro Universidad Santiago de Cali

https://orcid.org/0000-0002-8155-3277

Pregrado en fisioterapia. Correo: alejandro.gutierrez02@usc.edu.co

Cortes-Gómez Nicolás Universidad Santiago de Cali https://orcid.org/0000-0001-8656-2343

Pregrado en fisioterapia. Correo: nicolas.cortes00@usc.edu.co

Calero-Saa Pedro Antonio Universidad Santiago de Cali https://orcid.org/0000-0002-9978-7944

Fisioterapeuta, Especialista en Epidemiologia, Magister en Intervencion Integral en el Deportista. Docente de Tiempo Completo Universidad Santiago de Cali, programa de Fisioterapia, Coordinador Semillero Siafed, Investigador Grupo Salud y Movimiento. Correo: pedro.calero00@usc.edu.co 


\section{Cuadro y Gráficos}

\begin{tabular}{|c|c|c|c|c|c|c|}
\hline \multirow{2}{*}{$\mathbf{N}$} & \multirow{2}{*}{ PALABRAS CLAVE } & TOTAL & & & & \\
\hline & & PUBMED & SCIENCEDIRECT & SCOPUS & SCIELO & \\
\hline & $\begin{array}{l}\text { Riesgo cardiovascular } \\
\text { AND Adultos jóvenes }\end{array}$ & 11 & 977 & 33 & 1 & \\
\hline & $\begin{array}{l}\text { Obesidad AND Adultos } \\
\text { jóvenes }\end{array}$ & 2 & 870 & 32 & 2 & \\
\hline & $\begin{array}{l}\text { Adulto joven AND } \\
\text { Tabaquismo }\end{array}$ & 2 & 600 & 8 & 0 & \\
\hline & $\begin{array}{l}\text { Consumo de } \\
\text { alcohol AND Riesgo } \\
\text { cardiovascular }\end{array}$ & 5 & 2912 & 11 & 29 & \\
\hline & $\begin{array}{l}\text { Hábitos alimenticios } \\
\text { AND Adultos }\end{array}$ & 1 & 37 & 1 & 6 & \\
\hline & $\begin{array}{l}\text { Enfermedad } \\
\text { cardiovascular AND } \\
\text { Riesgo cardiovascular } \\
\text { AND adulto }\end{array}$ & 10 & 91 & 5 & 64 & \\
\hline & $\begin{array}{l}\text { Tabaquismo AND } \\
\text { Obesidad }\end{array}$ & 8 & 3736 & 20 & 52 & \\
\hline & $\begin{array}{l}\text { Consumo de alcohol } \\
\text { AND Tabaquismo. }\end{array}$ & 11 & 2328 & 21 & 81 & \\
\hline & TOTAL & 50 & 9274 & 131 & 235 & 9690 \\
\hline
\end{tabular}

Tabla 1. Proceso de búsquedas sin filtros

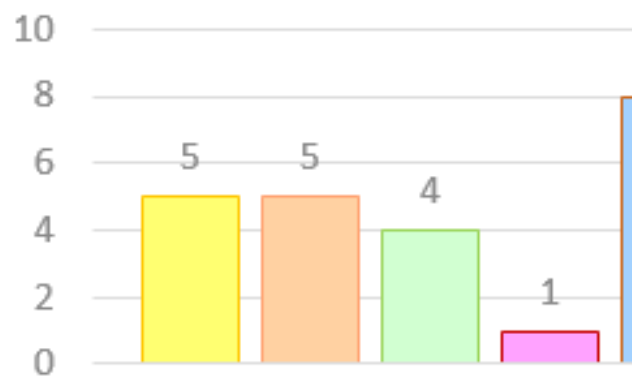

8

8
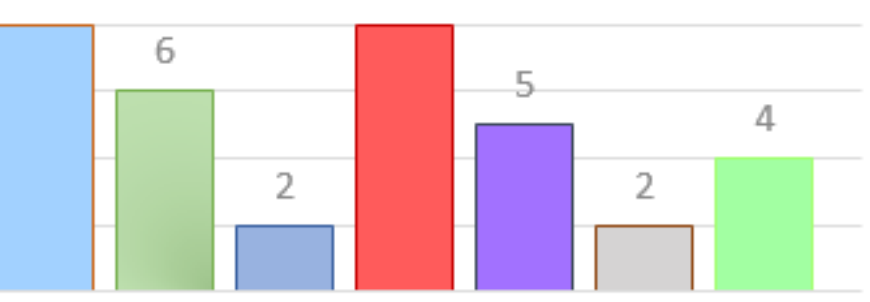

Año de publicación

$\square 2010 \square 2011 \square 2012 \square 2013 \square 2014 \square 2015$

$\square 2016 \square 2017 \square 2018 \square 2019 \square 2020$

llustración 1. Número de artículos por año de publicación de estudios seleccionados.

Fuente: elaboración propia. 

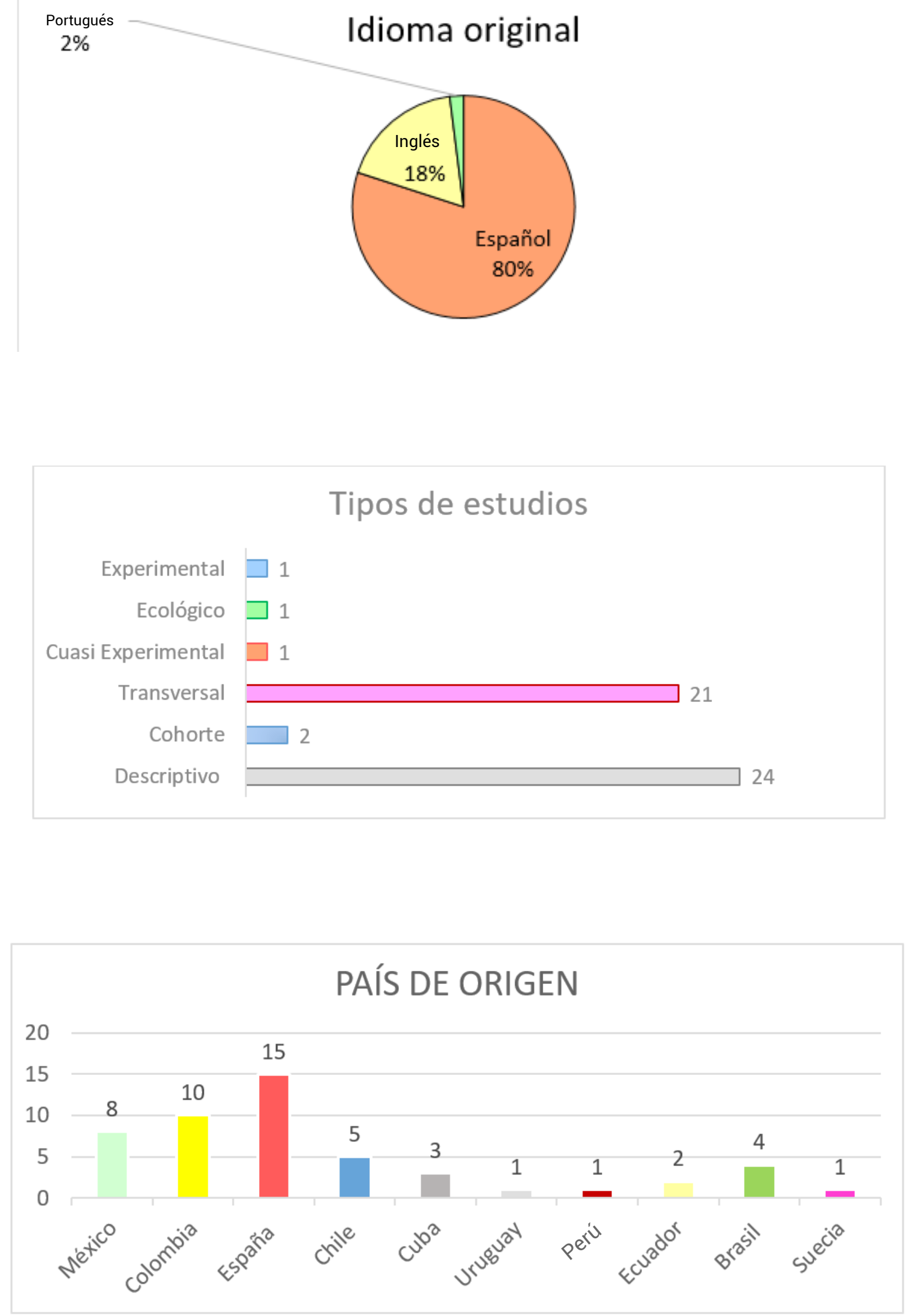